\title{
Influence of Standard Lightning Impulse Front Time Tolerances on the Flashover Voltage of Suspension Insulators
}

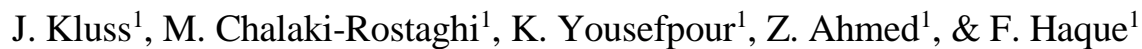 \\ ${ }^{1}$ Department of Electrical and Computer Engineering, Mississippi State University, Starkville, USA \\ joni@ece.msstate.edu
}

\begin{abstract}
For high voltage impulse testing, a standard lightning impulse is defined in IEEE Std. 4 and IEC 60060-1 as a double exponential waveform having a front time $T_{l}=1.2 \mu \mathrm{s} \pm 30 \%$ and time to half-value $T_{2}=50 \mu \mathrm{s} \pm 20 \%$. It has been noticed that for a given specimen, it is possible to successfully pass a flashover test at one end of the $T_{1}$ tolerance range while failing the same test at the opposite end of the tolerance spectrum. Consequently, a systematic approach was adopted to investigate this observation. Up-and-down tests were performed to define the disruptive discharge voltage (critical flashover voltage CFO, $U_{50}$ ) for $1,5,10$, and 15 unit glass insulator strings standard lightning impulses using the minimum acceptance front time value $\left(T_{1} \approx 0.84 \mu \mathrm{s}\right)$. Tests were repeated using the maximum tolerance value $\left(T_{1} \approx 1.56 \mu \mathrm{s}\right)$ to investigate the degree of divergence in the flashover value. Particular attention is given to the steepness (voltage-time characteristics) of the applied impulse to consider if tolerance criteria amendment is merited in a future standard revision. As the steepness impact is more renowned in non-uniform geometries, field homogeneity as a function of string length is also incorporated into the analysis.
\end{abstract}

\section{Introduction}

Standardization of high voltage test techniques is aimed at promoting the fulfilment of testing requirements. Through harmonization and compliance, the quality of testing (which is achieved through representativeness, repeatability, reproducibility, independence, and selectivity) is ensured. Standards (IEEE Std. 4, IEC 60060-1) define a lightning impulse voltages as a double exponential waveform having a front time $T_{1}=1.2 \mu \mathrm{s} \pm 30 \%(0.84 \ldots 1.56 \mu \mathrm{s})$ and time to half-value $T_{2}=$ $50 \mu \mathrm{s} \pm 20 \%(40 \ldots 60 \mu \mathrm{s})[1,2]$. This waveform has been determined to represent fast-front overvoltages caused by lightning. It is acknowledged that in practice lightning impulses vary in waveshape depending on widely ranging circumstances, but it is not practical to assess equipment against all potential forms of impulses. Moreover, as mentioned above, to ensure quality of testing and comparability of data, equipment must be tested with similar stresses, in a similar manner, under similar conditions.

According to historical reviews [3, 7], the need for a standard lightning impulse waveform was first expressed in the 1920s. The impulse waveform went through several different formats as more experimental data was acquired. IEEE initially recommended $0.5 / 5,1 / 10$, and $1.5 / 40 \mu \mathrm{s}$ time parameters in 1931 from which the latter was most widely utilized in USA. The $1.5 \mu \mathrm{s}$ front time was measured from zero point to crest $(0-$ $100 \%)$. Eventually the $10-90 \%$ front time calculation was adopted and front time changed to $1 \mu \mathrm{s}$, making the standard waveform 1/40. Around the same time, Germany issued their standard lightning impulse as 1/50 (also derived using the 10$90 \%$ method). In 1962, through collaboration between USA and Europe, IEC introduced the now familiar $1.2 / 50 \mu \mathrm{s}$ waveform obtained using 30-90\% coordinates to remove the influence of low voltage oscillations on front time calculations

Achieving the exact time parameters for the standard lightning impulse voltage waveform can be challenging as impulse sources, sample loading, and circuit configurations vary. As such, the standards provide tolerances expressing the acceptable amount of deviation from target values. If a test sample is stressed with the established standard waveform, within the specified tolerance range, and successfully meets the acceptance threshold for the given sample, it has successfully passed the test. However, is it possible for a sample to successfully pass an impulse voltage flashover test at one end of the tolerance range while failing the same test at the other end of the tolerance spectrum? This behavior was noticed at the Mississippi State University High Voltage Laboratory when evaluating long glass suspension insulator strings. Consequently, a systematic approach was adopted to identify the most influential factors.

\section{Test assembly and protocol}

The $50 \%$ disruptive discharge voltage ( $U_{50}$, critical flashover voltage $\mathrm{CFO}$ ) can be determined using the up-and-down method by applying voltage stress to the sample. The voltage level for each succeeding stress is either increased or decreased by $\Delta V$ according to the result of the previous stress - the voltage level is increase if no disruptive discharge occurs, or alternatively, the voltage level is decreased upon occurrence of disruptive discharge. $\Delta V$ should be $0.01 \ldots 0.03$ of $U_{50}$ and the number of useful applications $n \geq 20$. The $50 \%$ disruptive discharge voltage $U_{50}$ is given by

$$
U_{50}=\frac{\sum\left(k_{i} U_{i}\right)}{n}
$$

where $k_{i}$ is the number if stresses applied at the voltage level $U_{i}$. The first level of $U_{i}$ taken into account is that which at least two events (stresses) were applied. 


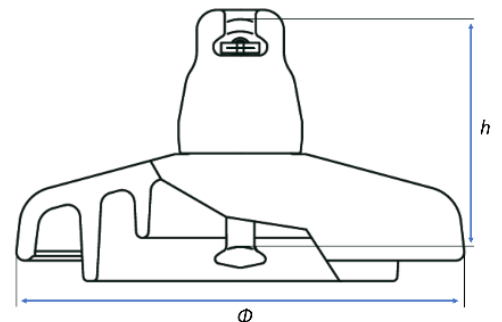

Figure 1. Toughened glass suspension insulator (fog type) used for measurements.

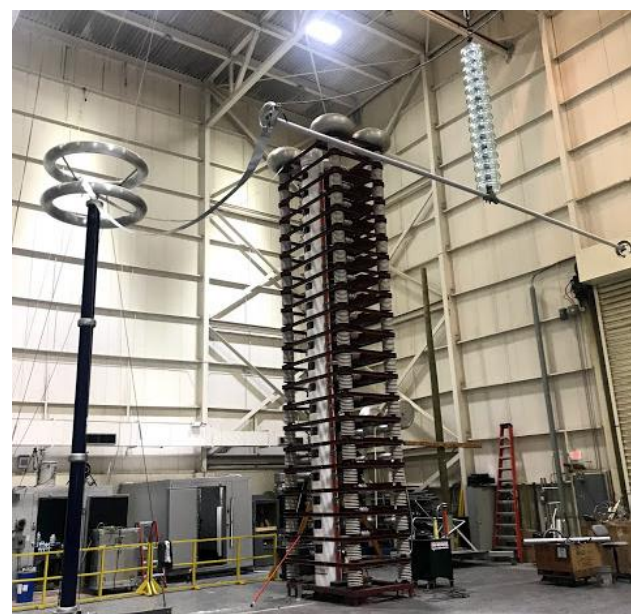

Figure 2. Test setup for up-and-down impulse measurements on glass insulator string.

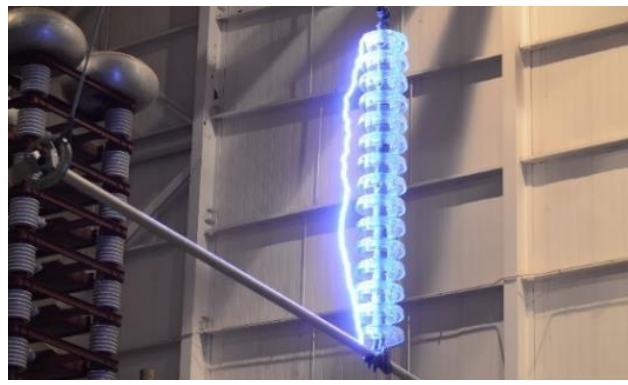

Figure 3. Flashover across 15 unit insulator string.

Test specimens were mounted according to ANSI/NEMA C29.1-2018 [4] requirements for suspension insulators. The tested toughened glass suspension insulators have a fog type profile (greater leakage distance due to long widely spaced under-ribs), diameter $\Phi=330 \mathrm{~mm}$, and spacing $h=146 \mathrm{~mm}$ as illustrated in Figure 1. Insulator strings consisting of 1, 5, 10, and 15 units were tested with both extremes of the front time range $\left(T_{l} \approx 0.84 \mu \mathrm{s}\right.$ and $\left.T_{l} \approx 0.1 .56 \mu \mathrm{s}\right)$ while maintaining a relatively constant $T_{2}$. High voltage lightning impulses were created using a $2.85 \mathrm{MV}, 50 \mathrm{~kJ}$, multistage Marx generator (Figure 2, Figure 3).

\section{Experimental results}

In total, 519 impulses where applied for the varying string lengths and voltage polarities. For the lower $T_{l}$ threshold margin, the target of $0.84 \mu \mathrm{s}$ was achieved with an average $T_{l}=0.87 \mu \mathrm{s}$ (standard deviation $\sigma=3 \%$ ). The upper margin of $1.56 \mu \mathrm{s}$ was achieved with an average of $T_{l}=1.56 \mu \mathrm{s}$ (standard deviation $\sigma$ $=3 \%$ ). The $50 \%$ disruptive discharge voltage $U_{50}$ was calculated using atmospheric correction factors and statistically treated

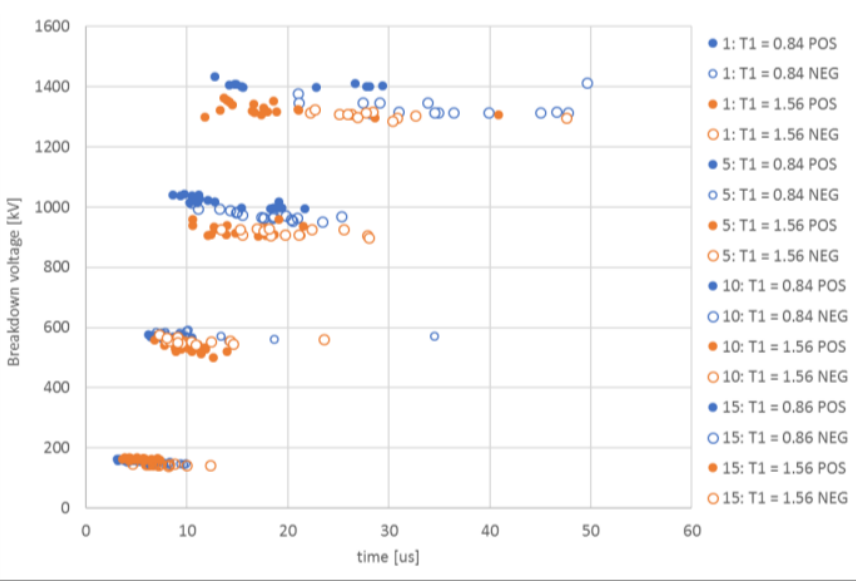

Figure 4. Measured flashover voltages (as a function of time-tochopping $T_{c}$ ) grouped by polarity and front time $T_{l}$.

Table 1. Measured flashover values.

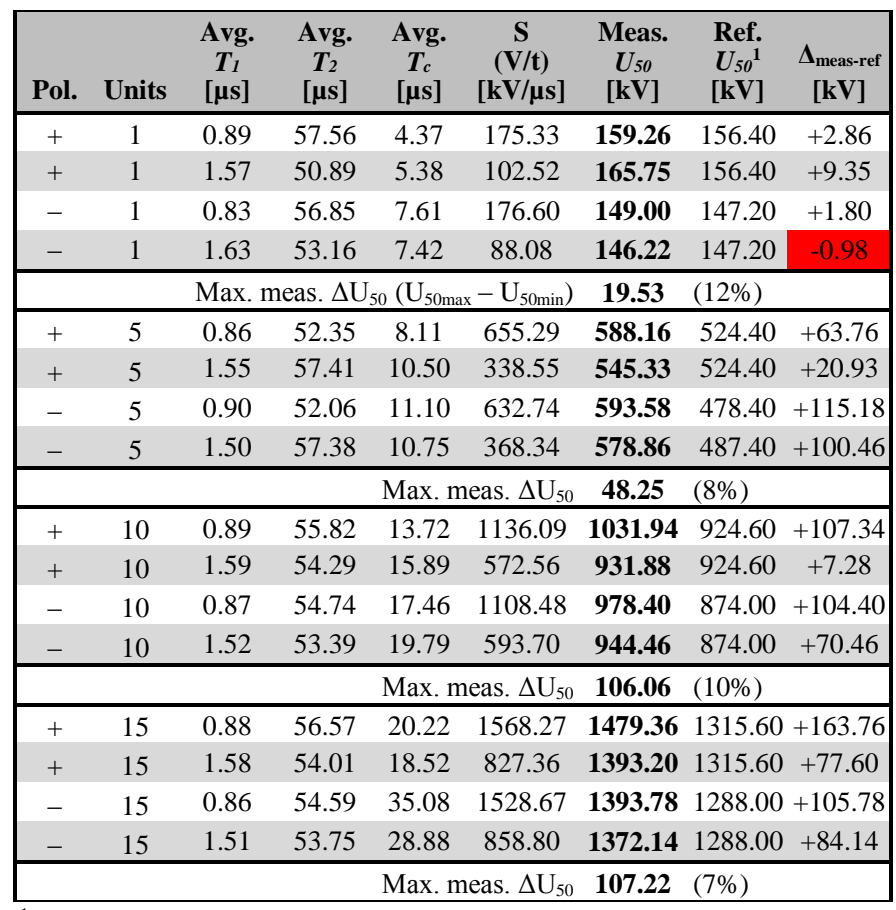

${ }^{1}$ Ref. $U_{50}$ values are $92 \%$ of the critical impulse flashover CIF values provided by the manufacturer specification sheet. Measured values should be equal to or exceed this $92 \%$ threshold value in order to meet acceptance criteria.

according to IEC 60060-1. The test voltage interval $\Delta V$ remained within the acceptable range, varying between $1.3 \ldots 2.8 \%$ of $U_{50}$ depending on the insulator string length (and hence, flashover voltage).

Measured data is presented in Figure 4 and Table 1. Measured $U_{50}$ values were compared with reference values obtained from manufacturer specifications (critical impulse flashover, CIF). All tested insulator string met the established acceptance criteria except the single unit string with negative polarity and maximum front time. However, in this particular case front time $T_{1}=1.63 \mu \mathrm{s}$ slightly exceeds the maximum allowable standard margin of $1.56 \mu \mathrm{s}$ and can thereby not be considered a strictly valid result. Although all other data falls within acceptable ranges, deviation for a given string length is large, measured $\Delta U_{50}$ varies $19 \ldots 107 \mathrm{kV}(7 \ldots 12 \%)$ depending on polarity and front time. For example, the 10-unit string clearly 
met acceptance criteria for negative polarity with both $T_{1}=$ $0.86 \mu \mathrm{s}$ and $1.51 \mu \mathrm{s}$, however, positive polarity results exceeded the acceptable limit by only $7 \mathrm{kV}$ when $T_{1}=1.59 \mu$ s (which can be considered a small margin).

Figure 4 suggests that higher breakdown voltages are achieved with the lower $T_{1}$ margin $(0.84 \mu \mathrm{s})$. The data implies that impulse tests utilizing these shorter front times result in larger passing margins compared to those utilizing front times of $1.56 \mu \mathrm{s}$. This translates into a larger probability of failure using slower front times in the range of $1.56 \mu \mathrm{s}$. This trend is most evident in the longer insulator strings (10 and 15 units). One could state that this behavior is due to the increased steepness $\mathrm{S}$ (voltage gradient, V/s), with smaller front times (higher steepness) resulting in higher breakdown voltages. This phenomenon is more prominent for non-uniform gaps as illustrated in Figure 5. This behavior, described using volt-time $(v-t)$ characteristic curves, is dependent on time lag (duration leading to breakdown upon application of sufficient voltage stress, Figure 6) and is specific to each gap geometry. Time lag consists of two components - statistical time lag $t_{s}$ and formative time lag $t_{f}$. Statistical time lag describes the time needed to produce the primary electron needed to initiate the ionization process (a statistical parameter dependent on gap length and ionization sources). Formative time lag is dependent on the discharge (ionization, transit time) mechanism and is also influenced by gap length. "The formative time lag increases with the gap length and the field nonuniformity, but it decreases with the applied overvoltage" [5].

This increase in formative time lag (and thus total time lag, time-to-chopping $T_{c}$ ) is clearly observed for the larger strings (refer to Figure 4). However, as displayed in Figure 7, the impact of steepness is not as clearly evident from the measured data. As front time $T_{l}$ is increased from $0.86 \mu$ s to $1.56 \mu \mathrm{s}$, steepness is approximately doubled. Doubling steepness has a limited impact on the breakdown voltage for the 1 and 5 unit strings. A slight trend is noticeable for the 10 and 15 unit strings where $U_{50}$ increases slightly for the higher steepness (lower $T_{l}$ ) data points. A similar trend was observed in [11] for long air gaps when $T_{1}$ was extended from 1.2 to $4.8 \mu \mathrm{s}-$ a longer $T_{l}$ results in a lower $U_{50}$ (c. 6\%). It is expected that larger steepnesses $(S>1600$ $\mathrm{kV} / \mu \mathrm{s}$ ) would exhibit a clearer trend. However, the up-and-down method results in tail-chopped pulses. In general, to observe a $v$ - $t$ characteristic curve, data is required from front chopped impulses where $T_{c} \approx 0.1 \ldots 0.5 \mu$ s produces $200 \ldots 300 \%$ flashover voltage values [6]. Such front times, however, are well below the allowable $0.84 \mu$ s tolerance range. Although relatively small, the steepness effect is most evident with positive polarity impulses, where doubling the steepness resulted in $U_{50}$ values increasing 5-10\% for 5, 10, and 15 unit strings but only $1.5-3.5$ $\%$ for the same insulator strings with negative polarity impulses. Thus, the impact of front time $T_{l}$ is most prominent for positive polarity impulses (varying $T_{l}$ results in largest divergence in $U_{50}$ ). However as seen in Figure 7 the impact of impulse polarity on the magnitude of breakdown voltage is inconsistent. For example, when compared to measured values with negative polarity, positive polarity impulses produces larger breakdown voltages for the 15-unit string whereas breakdown values with positive polarity are smaller for the 5-unit string (and the relationship is inconsistent for the 10-unit string).

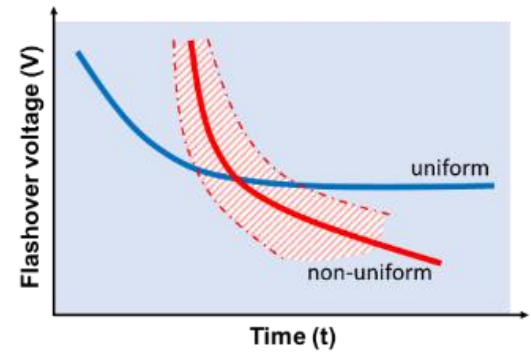

Figure 5. Volt-time $(v-t)$ characteristic curve for uniform and nonuniform fields.

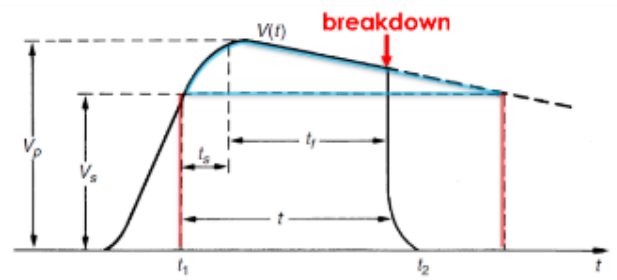

Figure 6. Time lag $t$ consisting of statistical time lag $t_{s}$ and formative time lag $t_{f}$. Edited from [5].

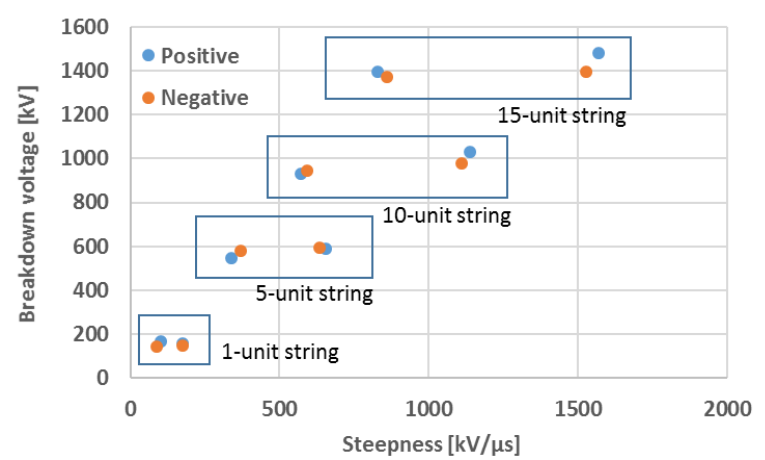

Figure 7. Breakdown voltage $U_{50}$ as a function of steepness.

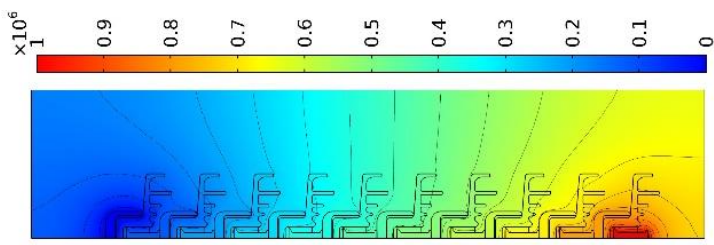

Figure 8. Voltage distribution along 10-unit string.

\section{Field uniformity}

Voltage is not distributed linearly across an insulator string. An uneven voltage distribution is unfavorable as it stresses certain units in the string more than others. Figure 8 displays the voltage distribution along the 10-unit insulator string. The insulator string can be viewed as a chain of capacitances. Each individual insulator unit in the string has its own self-capacitance $\Delta c_{s}$. In addition, each unit is influenced by stray capacitances (capacitance to grounded tower or cross arm, $\Delta c_{e}$, and capacitance to energized phase conductor, $\Delta c_{v}$ ) as illustrated in Figure 9. The voltage distribution depends on the capacitance ratios. Assuming unit capacitance $\Delta c_{e}$ and $\Delta c_{v}$ remain constant, unit capacitance can be related to string capacitance $C_{e}$ and $C_{v}$ by [6],

$$
\Delta c_{i}=\left(C_{i} / l\right) \Delta x=c_{i} \Delta x
$$

where $i=v$ or $e$ (denoting capacitance to phase conductor or 


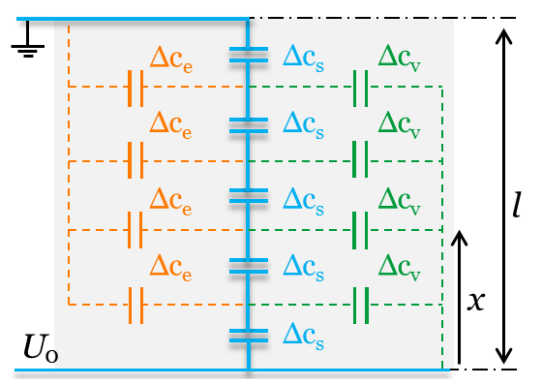

Figure 9. Capacitance chain formed by insulator units in a string.

grounded tower), $\Delta x$ is the length of the insulator unit and $l$ is the length of the insulator string. For the ratio $C_{v} / C_{e}=1$, voltage is distributed symmetrically. Voltage stress is concentrated on the grounded tower side when $C_{v} / C_{e}>1$, and in contrast when $C_{v} / C_{e}<1$, the voltage stress is focused on the energized conductor side. Non-uniformity of the voltage distribution increases with the increase in the string length (number of units in the insulator string).

Two-dimensional axisymmetric electrostatic finite element method (FEM) simulations were performed to investigate electric field and potential distributions of the investigated insulators strings. In a multiple unit insulator string, the cap of the top unit is defined as ground while the pin of the bottom unit is assigned an electric potential (equivalent to the CIF values for the corresponding string length). All other conducting components (caps and pins) were defined as floating potentials. The simulation calculates the degree of capacitive coupling to these floating potentials based on geometries, materials, and clearances. Higher electric field concentrations are observed where potentials are closest (shortest distances between cap and pin). Figure 10 plots the electric field intensity along the center axis of the string for the 15-unit insulator string. Electric field is highest at the ends of the string with maximum values concentrated around the conductor (high voltage) end of the string.

Assessing the field distribution along the center axis enables the formation of a uniformity factor describing the degree of electric field homogeneity along the string. Figure 11 shows the maximum electric field intensity along the center axis within each insulator unit for a given string (string length normalized to allow for improved visualization). The uniformity ratio $\eta=$ $E_{\min } / E_{\max }$ is $50 \%$ for the 5-unit string, $35 \%$ for 10 -units, and $32 \%$ for 15 units $(100 \%$ corresponds to a perfectly homogenous field). Uniformity along the center axis decreases as the insulator length increases. In all cases, field intensity is highest around the conductor end. However, as was visible in Figure 3, the discharge path of the flashover does not travel along the entire surface span of the insulators but instead bypasses the insulator string through the air (as is expected for clean dry samples). As such, the electric field distribution along such a path is of interest. Figure 12 shows the maximum electric field along the out diameter of the glass shell. Here, slightly higher field values are observed on the tower (ground) end of the string. The 5-unit string produces a near symmetrical field distribution as evident in the more detailed Figure 13. For 10 and 15 unit strings the distribution is slightly skewed with minimums approximately $65-70 \%$ of the distance from the grounded tower end. Despite slightly skewed distributions, overall uniformity is maintained

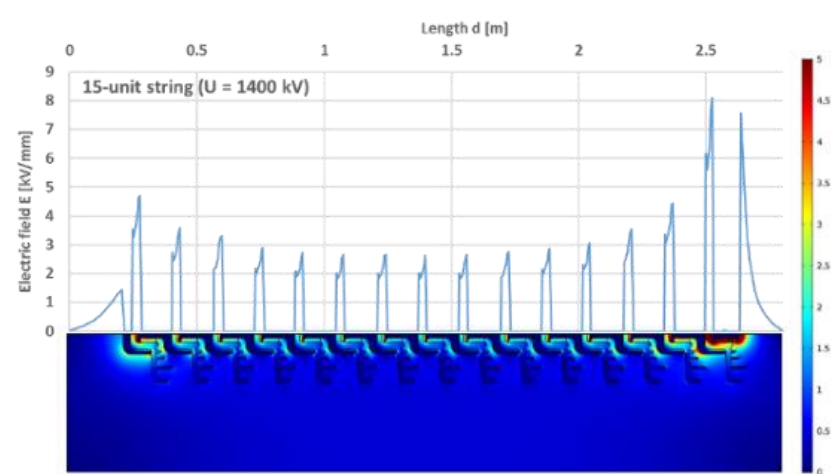

Figure 10. Electric field intensity along center axis of 15 -unit string.

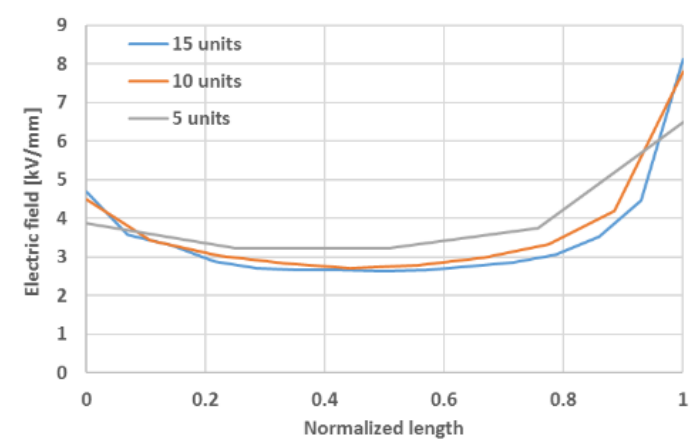

Figure 11. Maximum electric field distribution along center axis of insulator strings.

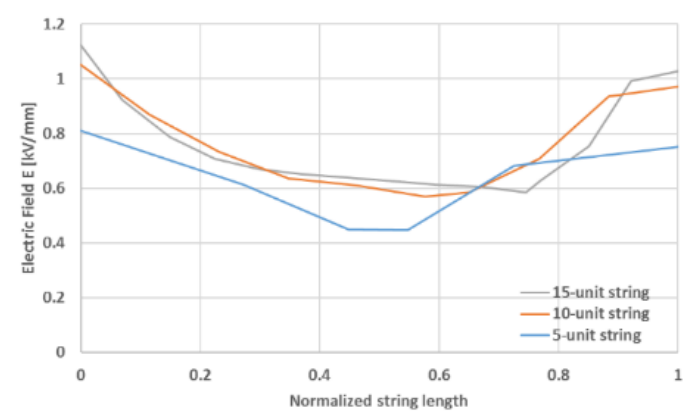

Figure 12. Maximum electric field distribution along outer diameter of insulator string shells.

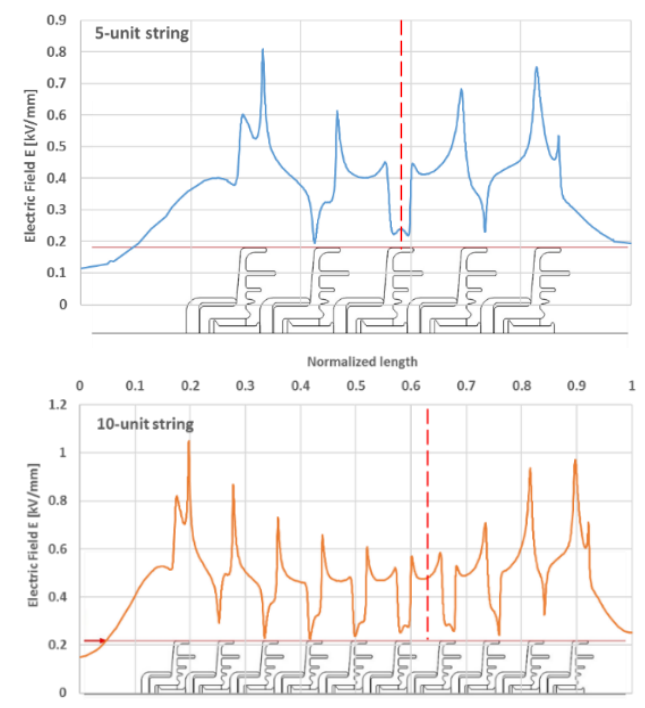

Figure 13. Electric field distribution along outer diameter of insulator shells (axis shown as red solid line) for 5-unit string (top) and 10-unit string (bottom). 
relatively constant for all strings along the outer shells, $\eta_{5-\text { unit }}=$ $55 \%, \eta_{10-\text { unit }}=54 \%, \eta_{15 \text {-unit }}=52 \%$.

In summation, voltage stress (electric field intensity) is highest around the conducting components on the conductor end of the insulator string, i.e., $C_{\mathrm{v}} / C_{\mathrm{e}}<1$. The field distribution in the air at the outer diameter of the insulator shells is rather symmetrical exhibiting slightly higher electric field values on the grounded tower end of the string. As expected, nonuniformity increase (symmetry becomes skewed) as insulator string length increases. However, does field distribution influence $U_{50}$ values? Observations by other researchers are very mixed for the relationship between $T_{1}$ and $U_{50}$ due to the large range of varying samples, materials, and experimental configurations [12-16]. In some cases, lower front times $T_{l}$ produce higher $U_{50}$ values, and in other cases the effect is opposite. If field homogeneity is taken as a common factor, according to [10], the impact of $T_{1}$ on $U_{50}$ is most prominent when field non-uniformity increases. The data in this research supports this statement that that $U_{50}$ is most influenced by $T_{1}$ for the longer strings which exhibit asymmetric and more nonuniform field distributions. However, this observation is valid mainly for positive polarity results and not as clearly evident for negative polarity data.

\section{Data validity}

If the fluctuation in measured $U_{50}$ is not clearly dominated by a physical phenomenon (polarity, uniformity, steepness), could it be influenced by the laboratory in which the data was acquired? All data was collected using the same measurement systems and measurement equipment. As such, any errors introduced by the system should be consistent for all of the measured values. The utilized voltage divider maintains linearity (within $0.4 \%$ ) throughout the voltage range investigated in this research. Thus, measured values for a single insulator unit should be as accurate as those recorded for the longer strings at higher voltages. However, one aspect which was not constant throughout the data collection period was atmospheric conditions. Measurements were performed in intervals over a period of 4 months during which seasonal changes resulted in fluctuation in ambient conditions.

According to IEC60060-1 and IEEE Std. 4 (Method 1), standard atmospheric conditions are defines as temperature $t_{0}=$ $20{ }^{\circ} \mathrm{C}$, absolute pressure $p_{0}=1013 \mathrm{hPa}$, and absolute humidity $h_{0}=11 \mathrm{~g} / \mathrm{m}^{3}$. The atmospheric correction factor $k$ consists of two components - air density correction factor $k_{1}$ and humidity correction factor $k_{2}\left(k=k_{1} k_{2}\right)$. Measured breakdown voltages $U$ under given test conditions are correlated to standard conditions by $U_{0}=U / k$. The applicability of the correction factor has limitations. The air density correction factor $k_{1}$ is considered reliable for $0.8<k_{1}<1.05$ and the absolute humidity $h$ to relative air density $\delta$ ratio is valid for $1 \mathrm{~g} / \mathrm{m}^{3}<h / \delta<20 \mathrm{~g} / \mathrm{m}^{3}$ for impulse voltages. Disruptive discharge voltage in air is expected to increase with an increase in either air density or humidity. However, this correlation becomes irregular when relative humidity exceeds $80 \%$ (in particular for disruptive discharge occurring over an insulating surface) [2]. IEC 60060-1 also includes a note that atmospheric corrections do not apply to flashover, only sparkover, where sparkover is defined as disruptive discharge occurring in gaseous (or liquid) dielectric while flashover is specified as disruptive discharge occurring over the surface of a dielectric in a gaseous or liquid media. As such, diverging procedures may be specified for specific apparatus.

For insulators, ANSI/NEMA C29.1 deviates from the horizontal standards IEC $60060-1$ by referring to IEEE Std. 4 Method 2 and by defining diverse standard conditions: temperature $t_{0}=25^{\circ} \mathrm{C}$ and absolute humidity $h_{0}=15 \mathrm{~g} / \mathrm{m}^{3}$, while absolute pressure $p_{0}$ remains the same at $1013 \mathrm{hPa}$. Measured values $U$ are corrected to standard conditions by $U_{0}=U /\left(k_{2} / k_{1}\right)$. Humidity correction factor $k_{2}$ is determined using atmospheric vapor pressure $P_{h}=0.0004615(h)(t+273)$ and the air density correction factor is calculated as $k_{l}=294(p) /(t+273)$. In IEEE Std. 4, Method 1 is perfectly harmonized with IEC60060-1 and is considered internationally the more accepted method of correcting voltages. However, Method 2, as described here, has been "a common method for historical testing and has value for tests on existing equipment designs" [1]. ANSI/NEMA C29.1 also requires volt-time curves to be corrected with full airdensity corrections as well as a varying humidity correction dependent on time-to-chopping $T_{c}$ - for flashover occurring at less than $10 \mu \mathrm{s}$, the correction shall be reduced by the direct ratio that the time to flashover bears to $10 \mu \mathrm{s}$. For the collected measurement data, such a requirement would apply to all 1-unit data. The consequences of such corrections are shown in Figure 14. Although a clear trend is evident, the corrections are extremely severe. E.g. raw data that fall into the $140-150 \mathrm{kV}$ breakdown range are corrected to values under $20 \mathrm{kV}$, which is not reasonable. As such, this time-to-chopping dependent correction factor is not applied to the acquired data and the IEC 60060-1 correction factors are considered more reliable than the ANSI/NEMA C29.1 corrections.

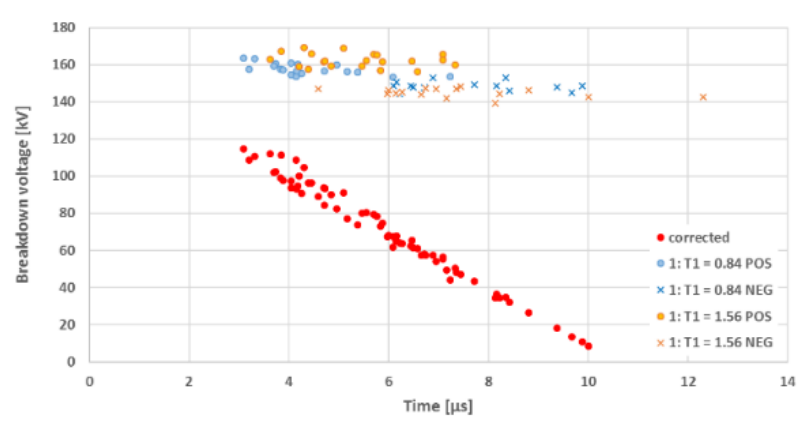

Figure 14. Influence of C29.1 volt-time correction factor on measured data of single unit string with $T_{c}<10 \mu \mathrm{s}$.

Table 2 presents and compares $U_{50}$ values derived using IEC60060-1/IEEE Std.4 Method 1 practices as well as procedures described in ANSI/NEMA C29.1 (Method 2), excluding the aforementioned time-to-chopping dependent humidity correction. For measurement data collected under the prevailing laboratory conditions, several circumstances failed to meet the specimen acceptances criteria (refer to uncorrected raw data in column $U_{50}[\mathrm{kV}]$ un-corr.). IEC/IEEE correction factors increased the passing rate by slightly adjusting measured values upward. In contrast, implementation of Method 2 specified in C29.1 results in reduced values and a majority of the test trials failing to meet the $92 \%$ CIF threshold value. 
Table 2. Influence of atmospheric correction factors on $U_{50}$

\begin{tabular}{|c|c|c|c|c|c|c|c|c|c|c|c|c|c|c|c|}
\hline \multirow[b]{2}{*}{ Pol. } & \multirow[b]{2}{*}{ Units } & \multirow{2}{*}{$\begin{array}{c}T_{l} \\
{[\mu \mathrm{s}]}\end{array}$} & \multirow{2}{*}{$\begin{array}{l}\text { RH } \\
{[\%]}\end{array}$} & \multirow{2}{*}{$\begin{array}{c}h \\
{\left[\mathrm{~g} / \mathrm{m}^{3}\right]}\end{array}$} & \multirow[b]{2}{*}{$h / \delta$} & \multirow{2}{*}{$\begin{array}{l}U_{50}[\mathrm{kV}] \\
\text { un-corr. }\end{array}$} & \multicolumn{3}{|c|}{ IEC/IEEE } & \multirow{2}{*}{$\begin{array}{c}U_{50}[\mathrm{kV}] \\
\text { IEC }\end{array}$} & \multicolumn{3}{|c|}{ ANSI/NEMA C.29.1 } & \multirow{2}{*}{$\begin{array}{c}U_{50}[\mathrm{kV}] \\
\mathrm{C} 29.1 \\
\end{array}$} & \multirow{2}{*}{$\begin{array}{c}\text { Ref. } U_{50} \\
(92 \% \text { CIF })\end{array}$} \\
\hline & & & & & & & $k_{1}$ & $k_{2}$ & $k=\left(k_{1}\right)\left(k_{2}\right)$ & & $k_{1}$ & $k_{2}$ & $k=k_{2} / k_{1}$ & & \\
\hline+ & 1 & 0.89 & 64 & 13.6 & 14.0 & 155.5 & 0.97 & N/A & 0.97 & 159.3 & 1.00 & 1.01 & 1.00 & 152.5 & 156.4 \\
\hline+ & 1 & 1.57 & 49 & 10.3 & 10.6 & 160.3 & 0.97 & N/A & 0.97 & 165.8 & 1.00 & 1.05 & 1.03 & 151.4 & 156.4 \\
\hline- & 1 & 0.83 & 38 & 7.4 & 7.5 & 147.0 & 0.99 & N/A & 0.99 & 149.0 & 0.99 & 1.06 & 1.06 & 139.0 & 147.2 \\
\hline- & 1 & 1.63 & 38 & 7.4 & 7.5 & 144.2 & 0.99 & N/A & 0.99 & 146.2 & 0.99 & 1.06 & 1.06 & 136.4 & 147.2 \\
\hline+ & 5 & 0.86 & 39 & 6.8 & 6.8 & 565.5 & 1.00 & 0.96 & 0.96 & 588.2 & 1.01 & 1.08 & 1.10 & 531.39 & 524.4 \\
\hline+ & 5 & 1.55 & 39 & 6.8 & 6.8 & 524.7 & 1.00 & 0.96 & 0.96 & 545.3 & 1.01 & 1.08 & 1.10 & 493.0 & 524.4 \\
\hline- & 5 & 0.90 & 38 & 6.3 & 6.3 & 565.9 & 1.00 & 0.95 & 0.95 & 593.6 & 1.02 & 1.08 & 1.10 & 533.4 & 478.4 \\
\hline- & 5 & 1.50 & 37 & 6.2 & 6.2 & 549.4 & 1.00 & 0.95 & 0.95 & 578.9 & 1.02 & 1.08 & 1.10 & 517.8 & 478.4 \\
\hline+ & 10 & 0.89 & 62 & 10.8 & 11.0 & 1005.0 & 0.98 & 1.00 & 0.98 & 1031.9 & 1.00 & 1.00 & 1.00 & 967.3 & 924.6 \\
\hline+ & 10 & 1.59 & 48 & 8.6 & 8.7 & 898.0 & 0.99 & 0.98 & 0.97 & 931.9 & 1.01 & 1.09 & 1.09 & 837.1 & 924.6 \\
\hline- & 10 & 0.87 & 62 & 10.9 & 11.0 & 961.5 & 0.98 & 1.00 & 0.98 & 978.4 & 1.00 & 1.04 & 1.04 & 924.4 & 874.0 \\
\hline- & 10 & 1.52 & 48 & 8.6 & 8.7 & 911.5 & 0.99 & 0.98 & 0.97 & 944.5 & 1.01 & 1.06 & 1.07 & 865.7 & 874.0 \\
\hline+ & 15 & 0.88 & 30 & 5.8 & 5.9 & 1390.9 & 0.99 & 0.95 & 0.94 & 1479.4 & 1.01 & 1.09 & 1.10 & 1284.5 & 1315.6 \\
\hline+ & 15 & 1.58 & 27 & 4.4 & 4.4 & 1311.4 & 1.01 & 0.93 & 0.94 & 1393.2 & 1.03 & 1.12 & 1.14 & 1206.0 & 1315.6 \\
\hline - & 15 & 0.86 & 30 & 5.8 & 5.9 & 1310.2 & 0.99 & 0.95 & 0.94 & 1393.8 & 1.01 & 1.08 & 1.09 & 1221.2 & 1288.0 \\
\hline- & 15 & 1.51 & 27 & 4.4 & 4.4 & 1292.0 & 1.01 & 0.93 & 0.94 & 1372.1 & 1.02 & 1.10 & 1.13 & 1203.9 & 1288.0 \\
\hline
\end{tabular}

\section{Conclusions}

The same samples were tested using the two extremes of front time $T_{1}$. For strings compromised on multiple units, the shorter $T_{1}=0.84 \mu$ s resulted consistently in larger breakdown voltages compared to $T_{1}=1.56 \mu$ s regardless of polarity. For longer insulator strings where field distribution is more asymmetric ( 10 and 15 unit strings), the highest $U_{50}$ values were obtained with a short $T_{1}$ and positive polarity. Interestingly, polarity influence is opposite to expectations. Traditionally, positive impulses produce significantly lower flashover values compared to negative polarity impulses [5, 9]. The results imply reduced passing margins for larger $T_{l}$ time parameters. Thus, to improve the probability of passing an impulse flashover test, the observed data suggests to use the shortest acceptable values for $T_{l}$. Properly designed products should withstand both normal operational stress as well as typical overvoltages. As such, it is important to test devices with representative voltage stresses. If testers start to intentionally favor shorter $T_{l}$ times $(<1 \mu \mathrm{s})$ in order to pass test requirements, is the procedure representative of natural lightning stress? Numerous authors [3, 7-8] have measured natural lightning waveforms with $T_{l}$ values ranging up to tens of microseconds and $T_{2}$ values extending to $200 \mu \mathrm{s}$ (observations vary based on conditions - distance to measurement location, voltage class, system conditions, etc.). To account for these longer time parameters, should positive tolerances for $T_{l}$ be extended further and negative tolerance be removed (e.g., $T_{l}=1.2 \mu \mathrm{s}+50 \%,-0 \%$ )? These are questions that should not be answered by one author or research group, but instead merits discussion in the appropriate standard committees. If tolerances are to be redefined, this would be most appropriate in apparatus specific standards which can account for typical loading of specific devices. Also, from all of the investigated variables, a highly influential factor appears to be related to post-processing of recorded data. As was demonstrated, implementation of varying correction factors can result in highly diverging results.

\section{References}

[1] IEEE Std 4-2013, "IEEE standard for high voltage testing techniques", 2013 [2] IEC 60060-1, "High-voltage test techniques - Part 1: General definitions and test requirements", $3^{\text {rd }}$ Ed., 2010

[3] W. Sima, P. Sun, M. Yang, J. Wu, J. Hua, "Impact of time parameters of lightning impulse on the breakdown characteristics of oil paper insulation", High Voltage, 1, (1), 2016, pp. 18-24, doi: 10.1049/hve.2016.0009

[4] ANSI/NEMA C29.1-2018, "American national standard for test methods for electrical power insulators", 2018

[5] E. Kuffel, W.S Zaengl, J. Kuffel, High voltage engineering fundamentals, Newnes, London, 2000

[6] M. Aro, J. Elovaara, M. Karttunen, K. Nousianen, V. Palva, Suurjännitetekniikka, Otatieto, Helsinki, 2015

[7] S. Okabe, J. Takami, T. Tsuboi, G. Ueta, A. Ametani, K. Hidaka, "Discussion on standard waveform in the lightning impulse voltage test," IEEE Trans. Dielectr. Electr. Insul., 20, (1), 2013, pp. 147-156, doi: 10.1109/TDEI.2013.6451353

[8] W. Lewis, C. Foust, "Lightning investigation on transmission lines - IV" Trans. AIEE, 53, (8), 1934, pp. 1180-1186, doi: 10.1109/TAIEE.1934.5056801

[9] T. Tsuboi, G. Ueta, S. Okabe, Y. Shimizu, T. Ishikura, E. Hino, "K-factor value and front-time-related characteristics in negative polarity lightning impulse test for UHV-class air insulation," IEEE Trans. Pow. Del., 28, (2), 2013, pp. 1148-1155, doi: 10.1109/TPWRD.2012.2235862

[10] T. Wen, Q. Zhang, L. Zhang, J. Ma, C. Guo, M. Chen, Y. Li, N. Shimomura, "Discussion on lightning impulse test waveform according to breakdown characteristics of $\mathrm{SF}_{6}$ gas gap, IEEE Trans. Dielectr. Electr. Insul., 24, (4), 2017, pp. 2306-2313, doi: 10.1109/TDEI.2017.006605

[11] S. Okabe, T. Tsuboi, G. Ueta, "Study on lightning impulse test waveform for UHV-class electric power equipment," IEEE Trans. Dielect. Electr. Insul., 19, (3), 2012, pp. 803-811, doi: 10.1109/TDEI.2012.6215083

[12]H. Ni, N. Guo, Y. Zhao, W. Yuan, X. Liu, Q. Zhang, T. Wang, "The effect of wavefront on the breakdown characteristics of transformer oil under positive impulse voltages" ICPADM, 2018, pp. 1074-1077, doi: 10.1109/ICPADM.2018.8401230

[13] B. Sharath, S. Usa, "Prediction of impulse voltage-time characteristics of air and oil insulation for different wavefronts", IEEE Trans. Dielectr. Electr. Insul, 16, (6), pp. 1693-1697, 2009, doi: 10.1109/TDEI.2009.5361591

[14] M. Wilson, I. Timoshkin, M. Given, S. Macgregor, T. Wang, "Breakdown of mineral oil: effect of electrode geometry and rate of voltage rise", IEEE Trans. Dielectr. Electr. Insul, 19, (5), pp. 1657-1664, 2012, doi: 10.1109/TDEI.2012.6311513

[15] F. Garnacho, E. Gockenbach, S. Berljin, P. Simon, K. Hackemack, P. Werle, "Evaluation of lightning impulse voltages based on experimental results proposal for the revision of IEC60060-1 and 61083-2", Electra, 204, pp. 3139, 2002.

[16] T. Tsuboi, G. Ueta, S. Okabe, Y. Shimizu, E. Hino "Insulation breakdown characteristics of UHV-class oil-immersed transformer for lightning impulse withstand voltage test waveform - K-factor value and front time related characteristics", IEEE Trans. Dielectr. Electr. Insul, 18, (6), pp. 2049-2058, 2011, doi: 10.1109/TDEI.2011.6118643 